\title{
Search for correlations between the BeppoSAX and RXTE GRBs locations and historical supernovae
}

\author{
F. Hroch, R. Hudec, and T. Rezek \\ Astronomical Institute, CZ-251 65 Ondřejov, Czech Republic
}

Received January 21; accepted May 10, 1999

\begin{abstract}
We have cross-correlated the precise positions of the recent GRBs obtained from the catalogue by Greiner (BeppoSAX and RXTE localizations) with the catalogue of historical supernovae provided by the Central Bureau for Astronomical Telegrams of the IAU.
\end{abstract}

Key words: gamma-ray bursts

\section{Introduction}

The appearance of the SN 1998bw (Sadler et al. 1998) in the close environment of GRB 980425 (Soffitta et al. 1998) implicates the question of possible association between supernovae and gamma-ray bursts. We have carried out a correlation for angular coincidences between the historical supernovae catalogue and recent precise observations of gamma-ray bursts with the main goal to search for possible recurrences of GRBs.

A study for temporal and angular coincidences with another set of precise GRBs positions has been carried out by Kippen et al. (1998) and by Wang \& Wheeler (1998). A detailed study of the supernova 1998bw and possible connections of SNe with GRBs has been presented by Woosley et al. (1999).

\section{Method and statistics}

We have searched for supernovae in 3-sigma error-box around the precise positions of the GRBs. The time correlation has been ignored due to search for possible recurrences of GRBs. This is an innovative approach different from studies by other authors (Kippen et al. 1998 and by Wang \& Wheeler 1998).

\subsection{The simple statistics of the precise localized GRBs}

- there are only 20 precisely localized GRBs by BeppoSAX and RXTE,

Send offprint requests to : R. Hudec
- the typical localization accuracy is $4^{\prime}$,

- these objects show non-isotropic distribution over the sky - only 6 from 20 GRBs have been found on the southern hemisphere. This distribution is caused by non-isotropic monitoring of the sky by the BeppoSAX satellite,

- catalogue source: Greiner 1998.

\subsection{The simple statistics of the supernovae catalogue}

- there are 1443 supernovae detected since 1885,

- non-isotropic distribution due to the galactic extinction and the observers preferences,

- localization precision better than $1^{\prime \prime}$,

- the supernova SN1998bw is not included,

- catalogue source: CBAT 1998.

\section{Conclusion}

No positive positional correlation between known supernovae and precise GRBs positions provided by BeppoSAX and RXTE has been found except the case of the SN 1998bw. However, it should be noted that this study is limited due to the large incompleteness of the supernovae catalogue.

Acknowledgements. We acknowledge J. Greiner and authors of the supernovae catalogue of the Central Bureau for Astronomical Telegrams for use of their products. The work has been supported by the Projects of the Czech Ministry for Education and Youth, Nos. ES02 and ES036.

\section{References}

Central Bureau for Astronomical Telegrams: 1998, http://cfa-www.harvard.edu/iau/lists/Supernovae.html

Greiner J., 1998, http://www.obs.aip.de/ jcg/

Kippen R.M., et al., 1998, ApJ 506, L27

Sadler E.M., Stathakis R., Cannon R., Galama T.J., 1998, IAU Circ. 6896

Soffitta P., et al., 1998, IAU Circ. 6884

Wang L., Wheeler J.G., 1998, ApJ 504, L87

Woosley S.E., Eastman R.G., Schmidt B.P., 1999, ApJ 516, 788 